\title{
SUMMABILITY AND UNIQUENESS OF DOUBLE TRIGONOMETRIC INTEGRALS
}

\author{
BY \\ VICTOR L. SHAPIRO
}

1. Introduction. Let $\phi$ be a complex-valued additive function of a set defined on the bounded Borel sets in the plane in the sense of Saks [7], and let $C_{1}+p$ designate the closed disc of radius 1 with center at $p$. Berkovitz [1] has shown that if the total variation $\int_{C_{1}+p}|d \phi(u)|=o\left(|p|^{-1}\right)$, then for any domain $D$ contained in the interior of the fundamental square $\Omega$ there is a double trigonometric series $\sum a_{m} e^{i m x}$ whose circular partial sums are uniformly equiconvergent with the circular partial integrals of $\int e^{i x u} d \phi(u)$ in $D$. In this paper we extend the results of Berkovitz to set functions whose total variation over unit circles with center $p$ is $o\left(|p|^{\alpha}\right), \alpha>-1$, and obtain results similar to his with equisummability $(C, \alpha+1)$ replacing equiconvergence. We then apply these results to the uniqueness of double trigonometric integrals and show that if $c(u)=o(|u|-\epsilon), \epsilon>0$, and if $\int_{E_{2}} e^{i x u} c(u) d u$ is $(C, 1)$ circularly summable to a function $f$ which satisfies a Lipschitz condition of order $\alpha$ on every bounded domain, then $\left(4 \pi^{2}\right)^{-1} \int_{E_{2}} e^{-i x u} f(x) d x$ is $(C, 1)$ circularly summable almost everywhere to $c(u)$. In particular if $f=0$, then $c(u)$ is almost everywhere equal to zero.

Zygmund's paper [10], where analogous results for the real line are obtained, provides the essential motivation for this paper.

2. Definitions and notations. As is well known, a complex-valued additive function of a set $\phi$ defined on the bounded Borel sets in the plane may be written in the form

$$
\phi=\phi_{1}-\phi_{2}+i\left(\phi_{3}-\phi_{4}\right),
$$

where $\phi_{i}(i=1, \cdots, 4)$ are non-negative additive functions of sets and represent respectively the upper variation and minus the lower variation of the real and imaginary parts of $\phi$ on any bounded Borel set. We shall designate the total variation of $\phi$ on the bounded Borel set $E$ by either $W(\phi, E)$ or $\int_{E}|d \phi(u)|$ and shall define it as the sum $\phi_{1}(E)+\phi_{2}(E)+\phi_{3}(E)+\phi_{4}(E)$.

The notation used in this paper will be vectorial; thus $u$ will designate the point with coordinates $\left(u_{1}, u_{2}\right), u x$ will be the scalar product, and $|u|$ will equal $\left(u_{1}^{2}+u_{2}^{2}\right)^{1 / 2}$.

By $f(u)=o\left(|u|^{\alpha}\right)$ will be meant: given an $\epsilon>0$ there is a $\left|u_{0}\right|$ such that if $|u|>\left|u_{0}\right|$, then $|f(u)|<\epsilon|u|^{\alpha}$.

By the set $E-u$ will be meant the set of points $\{x ; x=v-u, v$ in $E\}$.

Presented to the Society, April 26, 1952 and September 5, 1953; received by the editors July 13, 1953. 
$\bar{\alpha}$ and $[\alpha]$ shall designate respectively the smallest integer $\geqq \alpha$ and the largest integer $\leqq \alpha$.

The characteristic function of a set $E$ will be designated by $f_{E}(x)$.

By $\nabla^{2(t)}$, we shall mean the Laplacian operator $\left(D_{x_{1}}^{2}+D_{x_{2}}^{2}\right)$ iterated $t$ times. $\nabla^{2(0)}$ will be interpreted as the identity operator. $\Delta_{1} f(x)$ will designate the generalized Laplacian of $f$ at $x$ which is defined as $\alpha_{1}$ if

$$
(2 \pi)^{-1} \int_{0}^{2 \pi} f\left(x_{1}+r \cos \theta, x_{2}+r \sin \theta\right) d \theta=\alpha_{0}+\alpha_{1} r^{2} / 4+o\left(r^{2}\right) \quad \text { as } r \rightarrow 0 .
$$

The closed disc of radius $R$ will be designated by $C_{R}$ and the Euclidean plane by $E_{2}$. The fundamental square $\left\{x ;-\pi \leqq x_{i} \leqq \pi, i=1,2\right\}$ will be designated by $\Omega$.

We shall say that the trigonometric integral $\int_{E_{2}} e^{i x u} d \phi(u)$ is circularly summable $(C, \alpha), \alpha>0$, to the finite value $L(x)$ if the circular partial integrals of rank $R$,

$$
I_{R}(x)=\int_{C_{R}} e^{i x u} d \phi(u)
$$

are such that

$$
2 \alpha R^{-2 \alpha} \int_{0}^{R} I_{r}(x)\left(R^{2}-r^{2}\right)^{\alpha-1} r d r \rightarrow L(x) \quad \text { as } R \rightarrow \infty .
$$

All additive functions of a set introduced in the sequel, unless otherwise stated, will have as their domain the bounded Borel sets in the plane, will be countably additive, and, corresponding to Saks [7, p. 66], we shall call them, simply, additive functions of a set. All functions to be introduced will be Borel measurable, and unless otherwise stated the definitions and notations with regard to integration will be those of Saks [7].

3. Statement of main results. The main results of the paper are the following three theorems:

THEOREM 1. Let $T=\int_{E_{2}} e^{i x u} d \phi(u)$ be a double trigonometric integral with $W\left(\phi, C_{1}-u\right)=o\left(|u|^{\alpha}\right), \alpha>-1$. Let $D^{*}$ be a closed domain contained in the interior of the fundamental square $\Omega$. Then there exists a double trigonometric series $S=\sum a_{m} e^{i m x}$ with coefficients $a_{m}=o\left(|m|^{\alpha}\right)$ such that $\int_{C_{R}} e^{i x u} d \phi(u)$ $-\sum_{|m| \leqq R} a_{m} e^{i m x}$ is summable $(C, \alpha+1)$ to zero, uniformly for $x$ in $D^{*}$.

THEOREM 2. Let $T=\int_{E_{2}} e^{i x u} d \phi(u)$ be a double trigonometric integral with $W\left(\phi, C_{1}-u\right)=o\left(|u|^{\alpha}\right), \alpha>-1$. Let $D_{1}$ be a bounded closed domain. Then there exists a continuous function $g(u)=o\left(|u|^{\alpha}\right)$ such that $\int_{C_{R}} e^{i x u} d \phi(u)$ $-\int_{C_{R}} e^{i x u} g(u) d u$ is summable $(C, \alpha+1)$ to zero, uniformly for $x$ in $D_{1}$.

THEOREM 3. Let $c(u)$, in $L_{2}$ on any bounded domain, be $o(|u|-\epsilon), \epsilon>0$. Suppose the double trigonometric integral $\int_{E_{2}} e^{i x u} c(u) d u$ is circularly summable 
$(C, 1)$ to $f(x)$. Furthermore, suppose $f(x)$ is in Lip $\alpha$ on every bounded domain ( $\alpha$ positive and depending on the domain). Then the double trigonometric integral $\left(4 \pi^{2}\right)^{-1} \int_{E_{2}} e^{-i x u} f(x)$ is circularly summable $(C, 1)$ to $c(u)$ for almost every $u$.

Corollary. Let $c(u)$, in $L_{2}$ on any bounded domain, be $o\left(|u|^{-\epsilon}\right), \epsilon>0$. Suppose the double trigonometric integral $\int_{E_{2}} e^{i x u} c(u) d u$ is circularly summable $(C, 1)$ to zero for all values of $x$. Then $c(u)$ is equal to zero almost everywhere.

Berkovitz [1] proved Theorems 1 and 2 in the special case when $\alpha=-1$.

The hypothesis concerning $f$ in Theorem 3 can be weakened slightly, without changing the proof to be given, by assuming that $f$ satisfies a Morera condition instead of a Lipschitz condition. (For this, see Cheng [4, Theorem 3 ] where a uniqueness result analogous to the one presented here is obtained for double trigonometric series.)

To prove Theorem 1, it is first necessary to introduce the notions of the convolution of set functions and the formal product of trigonometric integrals.

4. Convolution of set functions and formal products of trigonometric integrals. Let $\phi$ and $\psi$ be two additive functions of a set. Then the set function $\chi$ defined by $\chi(A)=\int_{E_{2}} \phi(A-u) d \psi(u)$ for all bounded Borel sets $A$ is called the convolution of $\phi$ and $\psi$ and will be designated by $\phi * \psi$. The definition makes sense only if the above integral is defined. In particular we have the following lemma:

Lemma 1. Let $W\left(\phi, \quad C_{1}-u\right)=o\left(|u|^{k}\right), \quad k$ a real number, and let $\int_{E_{2}}\left(|u|^{k}+1\right)|d \psi(u)|<\infty$. Then $\chi=\phi * \psi=\psi * \phi$ is an additive function of $a$ set.

For let $A$ be any bounded Borel set. Then $|\phi(A-u)| \leqq W(\phi, A-u)$ $=o\left(|u|^{k}\right)$, and $\chi(A)$ is well defined.

To show that $\chi$ is an additive function of a set, take any sequence of disjoint sets $\left\{A_{i}\right\}$ contained in $A$. Then since

$$
W\left(\phi, \sum_{i=1}^{n} A_{i}-u\right) \leqq W\left(\phi, \sum_{i=1}^{\infty} A_{i}-u\right) \leqq W(\phi, A-u)=o\left(|u|^{k}\right),
$$

we have that $\chi\left(\sum_{i=1}^{\infty} A_{i}\right)$ is well defined and, furthermore, that

$$
\begin{aligned}
\chi\left(\sum_{i=1}^{\infty} A_{i}\right) & =\int_{E_{2}} \phi\left(\sum_{i=1}^{\infty} A_{i}-u\right) d \psi(u) \\
& =\int_{E_{2}} \sum_{i=1}^{\infty} \phi\left(A_{i}-u\right) d \psi(u)=\sum_{i=1}^{\infty} \chi\left(A_{i}\right),
\end{aligned}
$$

the last equality following from the Lebesgue dominated convergence theorem.

To show commutativity, we observe that $\int_{E_{2}} f_{A}(u+p)|d \phi(p)|$ $=W(\phi, A-u)=o\left(|u|^{k}\right)$, where $f_{A}(p)$ is the characteristic function of $A$, and 
consequently by Fubini that

$$
\int_{E_{2}} \phi(A-u) d \psi(u)=\int_{E_{2}} d \psi(u) \int_{E_{2}} f_{A}(u+p) d \phi(p)=\int_{E_{2}} \psi(A-p) d \phi(p) .
$$

Let $T_{1}=\int_{E_{2}} e^{i x u} d \phi(u)$ and $T_{2}=\int_{E_{2}} e^{i x u} d \psi(u)$ be two double trigonometric integrals with $\phi$ and $\psi$ as in Lemma 1 . We then define the formal product $T_{1} T_{2}=T_{3}$ to be the trigonometric integral $T_{3}=\int_{E_{2}} e^{i x u} d \chi(u)$ where $\chi=\phi * \psi$.

THEOREM 4. Let $T_{1}=\int_{E_{2}} e^{i x u} d \phi(u)$ and $T_{2}=\int_{E_{2}} e^{i x u} d \psi(u)$ be two double trigonometric integrals where $W\left(\phi, C_{1}-u\right)=o\left(|u|^{\alpha}\right), \alpha>-1$, and $\int_{E_{2}}|u|^{\delta}$ $\cdot|d \psi(u)|<\infty$ where $\delta=2 \bar{\alpha}+5$. Furthermore let $\lambda(x)=\int_{E_{2}} e^{i x u} d \psi(u)$ be such that

$$
D_{x_{1}}^{*} D_{x_{2}}^{t} \nabla^{2(z)} \lambda(x)=0
$$

for $x$ in $E$ and for non-negative integers $s, t$, and $z$ such that $0<s+t+z \leqq \bar{\alpha}+1$. Designate the formal product $T_{1} T_{2}$ by $T_{3}$. Then $T_{3}-\lambda T_{1}$ is circularly summable $(C, \alpha+1)$ to zero uniformly for $x$ in $E$.

REMark 1. Berkovitz [1, p. 351] proved this theorem using a different approach for $\alpha=-1$, where the derivative assumptions on $\lambda(x)$ are not necessary.

Set

$$
\chi=\phi * \psi \text {. }
$$

Then we see that the theorem will be proved when it is shown that

$$
\Delta_{R}(x)=\int_{C_{R}} e^{i x u} d \chi(u)-\lambda(x) \int_{C_{R}} e^{i x u} d \phi(u)
$$

is uniformly summable $(C, \alpha+1)$ to zero for $x$ in $E$. To do this, we first prove some lemmas.

LEMMA 2. Let $\phi_{x}$ and $\psi_{x}$ be additive functions of sets indexed by $x$ in $E$. Let $W\left(\phi_{x}, C_{1}-u\right)=o\left(|u|^{\alpha}\right)$ uniformly in $x$ and $\int_{E_{2}}|u|^{\delta}\left|d \psi_{x}(u)\right|<K$ where $K$ is a constant independent of $x$, and $\alpha$ and $\delta$ are as in Theorem 4. Furthermore let $\lambda_{x}(p)=\int_{E_{2}} e^{i p u} d \psi_{x}(u)$ be such that $D_{p_{1}}^{s} D_{p_{2}}^{t} \nabla^{2(z)} \lambda_{x}(0)=0$ for $0 \leqq s+t+z \leqq \bar{\alpha}+1$ where $s, t$, and $z$ are non-negative integers. Set $\chi_{x}=\phi_{x} * \psi_{x}$. Then $\chi_{x}\left(C_{R}\right)$ is uniformly summable $(C, \alpha+1)$ to zero for $x$ in $E$.

Observing that for fixed $u$,

$$
\begin{aligned}
\int_{0}^{R}\left(R^{2}-\rho^{2}\right)^{\alpha} \rho \psi_{x}\left(C_{\rho}-u\right) d \rho & =\int_{0}^{R} d \rho \int_{E_{2}}\left(R^{2}-\rho^{2}\right)^{\alpha} \rho f_{C_{\rho}}(p) d \psi_{x}(p-u) \\
& =(2 \alpha+2)^{-1} \int_{C_{R}}\left(R^{2}-|p|^{2}\right)^{\alpha+1} d \psi_{x}(p-u),
\end{aligned}
$$


we have that

$$
2(\alpha+1) \int_{0}^{R} \chi_{x}\left(C_{\rho}\right)\left(R^{2}-\rho^{2}\right)^{\alpha} \rho d \rho
$$

$$
=\int_{E_{2}} d \phi_{x}(u) \int_{C_{R}}\left(R^{2}-|p|^{2}\right)^{\alpha+1} d \psi_{x}(p-u) .
$$

Now in a manner similar to Berkovitz [2, p. 330], we shall show that the right side of (2) is $o\left(R^{2(\alpha+1)}\right)$, uniformly for $x$ in $E$.

Let $\alpha$ be a non-negative integer. Then $R^{2}-p^{2}=R^{2}-|u|^{2}+2 u_{1}\left(u_{1}-p_{1}\right)$ $+2 u_{2}\left(u_{2}-p_{2}\right)-|u-p|^{2}$ and

$$
\begin{aligned}
\left(R^{2}-p^{2}\right)^{\alpha+1}=\sum_{r+s+t+z=\alpha+1} \beta_{r, 8, t, z}\left[R^{2}-|u|^{2}\right] r|u-p|^{2 z} \\
\cdot\left[u_{1}\left(u_{1}-p_{1}\right)\right]^{*}\left[u_{2}\left(u_{2}-p_{2}\right)\right]^{t}
\end{aligned}
$$

where $\beta_{r, s, t, z}$ are constants and $r, s, t, z$ are non-negative integers. Since by hypothesis for fixed $u, \int_{E_{2}}|u-p|^{2 z}\left(u_{1}-p_{1}\right)^{s}\left(u_{2}-p_{2}\right)^{t} d \psi_{x}(p-u)=0$, we have if $|u| \leqq R-2$ that

$$
\begin{aligned}
\left|\int_{C_{R}}\right| u-\left.p\right|^{2 z}\left(u_{1}-p_{1}\right)^{s}\left(u_{2}-p_{2}\right)^{t} d \psi_{x}(p-u) \mid & \\
& \leqq \int_{E_{2}-C_{R-|u|}}|p|^{2 z}\left|p_{1}\right|^{s}\left|p_{2}\right|^{t}\left|d \psi_{x}(p)\right| \\
& \leqq K|R-| u||-(2 r+s+t+3)
\end{aligned}
$$

where $K$ is a constant independent of $u$ and $x$. Since a similar inequality holds if $R+2 \leqq|u| \leqq 2 R$, we have that

$$
\begin{aligned}
\left(\int_{C_{R}}\right. & \left.+\int_{C_{2 R}-C_{R+2}}\right)\left|d \phi_{x}(u)\right|\left(R^{2}-|u|^{2}\right)^{r}\left|u_{1}\right|^{*}\left|u_{2}\right|^{t} \\
& \cdot\left|\int_{C_{R}}\right| u-\left.p\right|^{2 z}\left(u_{1}-p_{1}\right)^{s}\left(u_{2}-p_{2}\right)^{t} d \psi_{x}(p-u) \mid \\
& \leqq 0\left[\left.R^{r}\left(\int_{C_{R-2}}+\int_{C_{2 R-C_{R+2}}}\right)\left|d \phi_{x}(u)\right||u|^{s+t}|R-| u\right|^{-8}\right] \\
& =o\left(R^{2(\alpha+1)}\right)
\end{aligned}
$$

uniformly in $x$.

It is furthermore clear that

$$
\left.\left.\int_{C_{R+2}-C_{R-2}}\left|d \phi_{x}(u)\right|\left|R^{2}-\right| u\right|^{2}|r| u\right|^{\diamond+t} \leqq o\left(R^{\alpha+1+r+s+t}\right)=o\left(R^{2(\alpha+1)}\right)
$$


uniformly in $x$, and that

$$
\int_{E_{2}-C_{2 R}}\left|d \phi_{x}(u)\right| \int_{C_{R}}\left(R^{2}-|p|^{2}\right)^{\alpha+1}\left|d \psi_{x}(p-u)\right|=o\left(R^{2(\alpha+1)}\right)
$$

uniformly in $x$.

Combining (3), (4), (5), and (6) we see from (2) that the lemma is proved in the special case when $\alpha$ is a non-negative integer.

When $W\left(\phi_{x}, C_{1}-u\right)=o\left(|u|^{\alpha+1}\right)$ uniformly in $x$ and $\alpha>-1$ is not an integer, we have after replacing $\alpha$ by $\bar{\alpha}$ in (3) and proceeding in the selfsame style as before that

$$
\int_{0}^{R} \chi_{x}\left(C_{\rho}\right)\left(R^{2}-\rho^{2}\right)^{\bar{\alpha}} \rho d \rho=o\left(R^{\alpha+\bar{\alpha}+2}\right)
$$

uniformly in $x$, and that

$$
\chi_{x}\left(C_{R}\right)=o\left(R^{\alpha+1}\right) \text { uniformly in } x .
$$

Writing the integral on the left side of (2) as the sum of the integrals

$$
\left[\int_{0}^{R-1}+\int_{R-1}^{R}\right] \chi_{x}\left(C_{\rho}\right)\left(R^{2}-\rho^{2}\right)^{\alpha} \rho d \rho=A+B,
$$

we see, after applying (7) and integrating by parts, that $A=o\left(R^{2(\alpha+1)}\right)$ uniformly in $x$. From (8), we see that $B$ also is $o\left(R^{2(\alpha+1)}\right)$ uniformly in $x$, which fact concludes the proof to the lemma.

Lemma 3. Let the hypotheses be the same as in Lemma 2 except that $\lambda_{x}(0)$ is not necessarily equal to zero. Then

$$
\Delta_{R, x}(0)=\chi_{x}\left(C_{R}\right)-\lambda_{x}(0) \phi_{x}\left(C_{R}\right)
$$

is uniformly summable $(C, \alpha+1)$ to zero for $x$ in $E$.

Noticing that $\psi_{x}\left(E_{2}\right)=\lambda_{x}(0)$, we define the additive function of a set $\psi_{2, x}$ in the following manner:

$$
\psi_{2, x}(A)= \begin{cases}\lambda_{x}(0) & \text { if } 0 \text { is in } A, \\ 0 & \text { if } 0 \text { is not in } A, \text { for any Borel set } A .\end{cases}
$$

Setting $\psi_{1, x}(A)=\psi_{x}(A)-\psi_{2, x}(A)$, it is then clear that

$$
\lambda_{1, x}(p)=\int_{E_{2}} e^{i u p} d \psi_{1, x}(u)=\lambda_{x}(p)-\lambda_{x}(0)
$$

and consequently that $\lambda_{1, x}$ satisfies the conditions of Lemma 2 . Therefore by that lemma $\chi_{1, x}\left(C_{R}\right)=\chi_{x}\left(C_{R}\right)-\chi_{2, x}\left(C_{R}\right)=\chi_{x}\left(C_{R}\right)-\lambda_{x}(0) \phi_{x}\left(C_{R}\right)$ is uniformly summable $(C, \alpha+1)$ to zero for $x$ in $E$ where $\chi_{i, x}=\phi_{x} * \psi_{i, x}, i=1,2$. 
We are now ready to prove the theorem, that is, to show that $\Delta_{R}(x)$ defined by (1) is uniformly summable $(C, \alpha+1)$ to zero for $x$ in $E$. To do this we define the following additive functions of sets:

$$
\phi_{x}(A)=\int_{A} e^{i x p} d \phi(p), \quad \psi_{x}(A)=\int_{A} e^{i x p} d \psi(p)
$$

for all $x$ in $E$ and then observe that

$$
\lambda(x)=\int_{E_{2}} e^{i x u} d \psi(u)=\int_{E_{2}} d \psi_{x}(u)=\lambda_{x}(0), \quad \lambda(x+p)=\lambda_{x}(p) \quad \text { for all } p .
$$

Since $\phi_{x}, \psi_{x}$, and $\lambda_{x}$ satisfy the hypotheses of Lemma 3, we have by that lemma that

$$
\chi_{x}\left(C_{R}\right)-\lambda_{x}(0) \phi_{x}\left(C_{R}\right)=\chi_{x}\left(C_{R}\right)-\lambda(x) \int_{C_{R}} e^{i x u} d \phi(u)
$$

is uniformly summable $(C, \alpha+1)$ to zero for $x$ in $E$. If it can be shown that $\chi_{x}\left(C_{R}\right) \equiv \phi_{x} * \psi_{x}\left(C_{R}\right)=\int_{C_{R}} e^{i x u} d \chi(u)$, then $\Delta_{R}(x)$, defined by (1), is equal to the right side of (9) and the theorem is proved. We shall now show this latter fact.

Let $A$ be any bounded Borel set and $x$ any fixed point in $E$. Choose a sequence of simple functions $\left\{g_{n}(u)\right\}$ such that $\left|g_{n}(u)\right| \leqq 1$ and such that $g_{n}(u) \rightarrow e^{i x u}$ for all $u$. Then noticing that $\left|\int_{A} g_{n}(u) d \phi(u-p)\right| \leqq W(\phi, A-p)$, which is an integrable function with respect to $\psi$, we have

$$
\begin{aligned}
\int_{A} e^{i x u} d \chi(u) & =\lim _{n \rightarrow \infty} \int_{E_{2}}\left[\int_{A} g_{n}(u) d \phi(u-p)\right] d \psi(p) \\
& =\int_{E_{2}}\left[\int_{A} e^{i x(u-p)} d \phi(u-p)\right] e^{i x p} d \psi(p) \\
& =\int_{E_{2}} \phi_{x}(A-p) d \psi_{x}(p) \\
& =\phi_{x^{*}} \psi_{x}(A),
\end{aligned}
$$

which concludes the proof to the theorem.

5. Formal differentiation. Let $T$ be the double trigonometric integral $\int_{E_{2}} e^{i x u} d \phi(u)$. Then by $\left(\nabla^{2(z)} D_{x_{1}}^{s} D_{x_{2}}^{z}\right) T$ is meant the trigonometric integral which one obtains by applying the operator formally to $T$. Thus

$$
\left(\nabla^{2(z)} D_{x_{1}}^{s} D_{x_{2}}^{t}\right) T=\int_{E_{2}} e^{i x u} d \phi_{z, s, t}(u)
$$

where 


$$
\phi_{z, s, t}(A)=\int_{A}(i)^{2 z+s+t}|u|^{2 z} u_{1}^{s} u_{2}^{t} d \phi(u)
$$

for all bounded Borel sets $A$. In a similar manner we define the sum of two trigonometric integrals and the product of a constant and a double trigonometric integral. Finally we shall say that $\int_{E_{2}} e^{i x u} d \theta(u)=\int_{E_{2}} e^{i x u} d \phi(u)$ if $\theta(A)$ $=\phi(A)$ for all bounded Borel sets $A$.

Let $\omega$ be a positive integer. Then since $|u|^{2}=|u-x|^{2}+2 x_{1}\left(u_{1}-x_{1}\right)$ $+2 x_{2}\left(u_{2}-x_{2}\right)+|x|^{2}$, we see that

$$
|u|^{2 \omega}=\sum_{r+s+t+z=\omega} \rho_{r, s, t, 2}|u-x|^{2 r}|x|^{2 z}\left[x_{1}\left(u_{1}-x_{1}\right)\right]^{s}\left[x_{2}\left(u_{2}-x_{2}\right)\right]^{t}
$$

where $\rho_{r, 8, t, z}$ are constants such that $\rho_{r, s, t, z}=\rho_{z, s, t, r}$ for non-negative integers $r, s, t, z$.

LEMMA 4. Let $T_{1}=\int_{E_{2}} e^{i x u} d \phi(u)$ and $T_{2}=\int_{E_{2}} e^{i x u} d \psi(u)$ where $W\left(\phi, C_{1}-u\right)$ $=o(1)$ and $\int_{E_{2}}|u|^{k} d \psi(u)<\infty$ where $k \geqq 2$. Set $\omega=[k / 2]$. Then the formal product $P=T_{1} T_{2}$ exists and

$$
\nabla^{2(\omega)} P=\sum_{r+8+t+z=\omega} \rho_{r, 8, t, z}\left[\left(\nabla^{2(z)} D_{x_{1}}^{s} D_{x_{2}}^{t}\right) T_{1}\right]\left[\left(\nabla^{2(r)} D_{x_{1}}^{8} D_{x_{2}}^{t}\right) T_{2}\right]
$$

where $\rho_{r, 8, t, z}$ are defined by (11).

Setting $\chi=\phi * \psi$, we see by Lemma 1 that $P$ exists and that $\nabla^{2(\omega)} P$ $=\int_{E_{2}} e^{i x u} d \widehat{\chi}(u)$ where $\widehat{\chi}(A)=\int_{A} i^{2 \omega}|u|^{2 \omega} d \chi(u)$. Defining $\phi_{z, 8, t}$ and $\psi_{r, 8, t}$ as in (10) and setting $\chi_{r, s, t, z}=\phi_{z, s, t} * \psi_{r, s, t}$, which exists by Lemma 1 , we see that the lemma will be proved when it is shown that

$$
\widehat{\chi}(A)=\sum_{r+8+t+z=\omega} \rho_{r, s, t, z} \chi_{r, 8, t, z}(A)
$$

for all bounded Borel sets $A$.

Now

$$
\begin{aligned}
& \chi_{r, 8, t, z}(A)= i^{2 \omega} \int_{E_{2}}|u|^{2 r} u_{1}^{s} u_{2}^{t} d \psi(u) \int_{E_{2}} f_{A}(u+p)|p|^{2 z} p_{1}^{s} p_{2}^{t} d \phi(p) \\
&=i^{2 \omega} \int_{E_{2}} d \psi(u) \int_{E_{2}} f_{A}(p)|u|^{2 r}|u-p|^{2 z} \\
& \cdot\left[u_{1}\left(p_{1}-u_{1}\right)\right] \cdot\left[u_{2}\left(p_{2}-u_{2}\right)\right] t d \phi(p-u)
\end{aligned}
$$

and consequently, from (11),

$$
\sum \rho_{r, 8, t, z} \chi_{r, s, t, z}(A)=(i)^{2 \omega} \int_{E_{2}} d \psi(u) \int_{A}|p|^{2 \omega} d \phi(p-u)
$$

for any bounded Borel set $A$. 
Choosing a sequence of simple functions $\left\{g_{n}(u)\right\}$ such that $g_{n}(u) \rightarrow|u|^{2 \omega}$ uniformly on every finite interval, we have that

$$
\begin{aligned}
\widehat{\chi}(A)=(i)^{2 \omega} \int_{A}|u|^{2 \omega} d \chi(u) & =(i)^{(2 \omega)} \lim _{n \rightarrow \infty} \int_{E_{2}} d \psi(p) \int_{A} g_{n}(u) d \phi(u-p) \\
& =(i)^{(2 \omega)} \int_{E_{2}} d \psi(u) \int_{A}|p|^{2 \omega} d \phi(p-u) .
\end{aligned}
$$

(12) then follows from (13) and (14), and the lemma is proved.

6. Localization. To the double trigonometric integral $T=\int_{E_{2}} e^{i x u} d \phi(u)$ where $W\left(\phi, C_{1}-u\right)=o\left(|u|^{\alpha}\right), \alpha>-1$, we associate the Riemann function

$$
\begin{aligned}
F(x)= & \phi(0)\left(x_{1}+x_{2}\right)^{2 \omega} /(2 \omega) ! 2^{\omega}+\int_{C_{1 / 2}-0} L(x, u) d \phi(u) \\
& +(-1)^{\omega} \int_{E_{2}-C_{1 / 2}} e^{i x u}|u|^{-2 \omega} d \phi(u)
\end{aligned}
$$

where $\omega=[\alpha / 2]+2$ and

$$
L(x, u)=\frac{e^{i x u}-\left[1+(i x u) / 1 !+(i x u)^{2} / 2 !+\cdots+(i x u)^{2 \omega-1} /(2 \omega-1) !\right]}{(i)^{2 \omega}|u|^{2 \omega}}, \quad u \neq 0 .
$$

It is clear that $\nabla^{2}$ applied formally $\omega$ times to $F(x)$ will give back the integral $T$. Furthermore since $2 \omega-\alpha>2$, it is also clear that the last integral on the right in (15) converges uniformly in $x$.

Given two closed bounded domains $D_{1}$ and $D$, it is well known that if $D_{1} \subset D^{0}$, the interior of $D$, a function $\lambda(x)$ of class $C^{(k)}$ can be constructed for any non-negative integer $k$ such that $\lambda(x)=1$ if $x$ is in $D_{1}$ and $=0$ if $x$ is not in $D$. We shall call such a function $\lambda$ a localizing function for the domains $D_{1}$ and $D$.

Setting $D_{R}(x)=\int_{C_{R}} e^{i x u} d u$, we obtain the following localizing theorem.

THEOREM 5. Let $T=\int_{E_{2}} e^{i x u} d \phi(u)$ where $W\left(\phi, C_{1}-u\right)=o\left(|u|^{\alpha}\right), \alpha>-1$. Let $\lambda(x)$ be the localizing function of class $C^{[2(\delta+\omega)]}$ for the closed bounded domains $D_{1}$ and $D$ where $D_{1} \subset D^{0}, \omega=[\alpha / 2]+2$, and $\delta=2 \bar{\alpha}+5$. Furthermore let $F(x)$ be the Riemann function of $T$. Then the difference

$$
\Delta_{R}(x)=\int_{C_{R}} e^{i x \omega} d \phi(u)-\left(4 \pi^{2}\right)^{-1} \int_{E_{2}} F(u) \lambda(u) \nabla^{2(\omega)} D_{R}(x-u) d u
$$

is uniformly summable $(C, \alpha+1)$ to zero for $x$ in $D_{1}$.

From the decomposition of $\phi$ mentioned at the beginning of $\$ 2$, we see that there is no loss in generality if we assume $\phi$ to be a non-negative additive function of a set. The proof of the theorem will be given in three parts. For the first part, we shall assume $\phi\left(C_{1 / 2}\right)=0$. Then designating the set function 
$\int_{A}(-1)^{\omega}|u|-{ }^{2 \omega} d \phi(u)$ defined on all the Borel sets in the plane by $\Phi(A)$, we have that

$$
F(x)=\int_{E_{2}-C_{1 / 2}} e^{i x u} d \Phi(u)=T_{\omega} .
$$

Set $\gamma(u)=\left(4 \pi^{2}\right)^{-1} \int_{E_{2}} e^{-i x u} \lambda(x) d x$. Then since $\lambda(x)$ is of class $C^{[2(\delta+\omega)]}$, $\gamma(u)=\left(4 \pi^{2}\right)^{-1} \int_{E_{2}} e^{-i x u}(-i|u|)^{-2(\delta+\omega)} \nabla^{2(\delta+\omega)} \lambda(x) d x$ if $u \neq 0$, and consequently $\gamma(u)=o\left(|u|^{-2(\delta+\omega)}\right)$ and is in $L_{2} \cap L_{1}$ on the plane. Therefore

$$
\lambda(x)=\int_{E_{2}} \gamma(u) e^{i x u} d u=\int_{E_{2}} e^{i x u} d \Gamma(u)=\mathfrak{F}[\lambda]
$$

where $\mathfrak{F}[\lambda]$ denote the Fourier integral of $\lambda$ and $\Gamma(A)=\int_{A} \gamma(u) d u$ for Borel sets $A$. Furthermore it is clear that if $r+s+t \leqq \omega$, then

$$
\mathfrak{F}\left[\left(\nabla^{2(r)} D_{x_{1}}^{8} D_{x_{2}}^{t} \lambda(x)\right]=\left(\nabla^{2(r)} D_{x_{1}}^{8} D_{x_{2}}^{t}\right) \mathfrak{F}[\lambda]\right.
$$

and also that

$$
\int_{E_{2}}|u|^{2 \omega+1}|d \Gamma(u)|<\infty .
$$

This latter fact in conjunction with the observation that $W\left(\Phi, C_{1}-u\right)$ $=o\left(|u|-{ }^{(2+\nu)}\right), \nu>0$, gives from Lemma 4 that

$$
\nabla^{2(\omega)}\left(T_{\omega} \mathfrak{F}[\lambda]\right)=\sum_{r+8+t+z=\omega} \rho_{r, 8, t, z}\left(\nabla^{2(z)} D_{x_{1}}^{s} D_{x_{2}}^{t}\right) T_{\omega}\left(\nabla^{2(r)} D_{x_{1}}^{s} D_{x_{2}}^{t}\right) \mathfrak{F}[\lambda]
$$

By (17), unless $r=s=t=0,\left(\nabla^{2(r)} D_{x_{1}}^{s} D_{x_{2}}^{t}\right) \mathfrak{F}[\lambda]$ converges uniformly to zero for $x$ in $D_{1}$, and consequently the circular partial integrals of every formal product in the sum on the right in (18) are uniformly summable $(C, \alpha+1)$ to zero for $x$ in $D_{1}$ by Theorem 4 . Observing from $(10)$ that $\rho_{0,0,0, \omega}=1$, we conclude that the circular partial integrals of $\nabla^{2(\omega)}\left(T_{\omega} \mathfrak{F}[\lambda]\right)-\left(\nabla^{2(\omega)} T_{\omega}\right) \mathfrak{F}[\lambda]$ are uniformly summable $(C, \alpha+1)$ to zero for $x$ in $D_{1}$. But $\nabla^{2(\omega)} T_{\omega}=T$, and by Theorem $4, T \mathfrak{F}[\lambda]-\lambda T$ is uniformly circularly summable $(C, \alpha+1)$ to zero for $x$ in $D_{1}$. So we can conclude further that the circular partial integrals of

$$
\nabla^{2(\omega)}\left(T_{\omega} \mathfrak{F}[\lambda]\right)-\lambda T
$$

are uniformly summable $(C, \alpha+1)$ to zero for $x$ in $D_{1}$. Since for $x$ in $D_{1}, \lambda(x)$ $=1$, to conclude the proof of the theorem in this first case where $\phi\left(C_{1 / 2}\right)=0$, it only remains to show that the circular partial integral of rank $R$ of $\nabla^{2(\omega)}\left(T_{\omega} \mathfrak{F}[\lambda]\right)$ in (19) is equal to

$$
\left(4 \pi^{2}\right)^{-1} \int_{E_{2}} F(u) \lambda(u) \nabla^{2(\omega)} D_{R}(x-u) d u
$$

in (10). This will now be done. 
Setting $P=T_{\omega} \mathfrak{F}[\lambda]$ and $M=\Gamma * \Phi$, observing that $\Phi$ is a positive or negative measure (according to the sign of $(-1)^{\omega}$ ) defined on the additive class of Borel sets in the plane and that $W(\Gamma, A)=0$ for all sets $A$ in this class of Lebesgue measure zero, we conclude that $M(E)=\int_{E} \mu(u) d u$ for Borel sets $E$ where $\mu$ is in $L_{1}$ on the plane and that

$$
P=\int_{E_{2}} e^{i x u} \mu(u) d u .
$$

By Remark 1, the circular partial integral of $P-\lambda T_{\omega}$ converges to zero uniformly in $x$; consequently

$$
\int_{E_{2}} e^{i x u} \mu(u) d u=\lambda(x) F(x) \quad \text { (which equals zero when } x \text { is not in } D \text { ) }
$$

and

$$
\mu(u)=\left(4 \pi^{2}\right)^{-1} \int_{E_{2}} e^{-i x u} F(x) \lambda(x) d x .
$$

It then follows that the circular partial integral of rank $R$ of $\nabla^{2(\omega)} P$ is

$$
\begin{aligned}
\int_{C_{R}} e^{i x \omega}(-1)^{\omega}|u|^{2 \omega} \mu(u) d u & =\left(4 \pi^{2}\right)^{-1} \int_{E_{2}} F(p) \lambda(p) d p \int_{C_{R}} e^{i u(x-p)}(-1)^{\omega}|u|^{2 \omega} d u \\
& =\left(4 \pi^{2}\right)^{-1} \int_{E_{2}} F(p) \lambda(p) \nabla^{2(\omega)} D_{R}(x-p) d p,
\end{aligned}
$$

which concludes the first part of the proof to this theorem.

REMARK 2. It is apropos at this time to point out that, using (19), we have just proved

$$
\int_{C_{R}} e^{i x u}(-1)^{\omega}|u|^{2 \omega} \mu(u) d u-\int_{C_{R}} e^{i x u} d \phi(u)
$$

is uniformly summable $(C, \alpha+1)$ to zero for $x$ in $D_{1}$ in the case $W\left(\phi, C_{1 / 2}\right)=0$. In Lemma 5 it will be shown that $\mu(u)$ is continuous and $\mu(u)=o\left(|u|^{\alpha-2 \omega}\right)$.

For the second part of the proof, we assume that $\phi\left(E_{2}-C_{1 / 2}\right)$ and that $\phi(0)=0$. Consequently by (13)

$$
F(x)=\int_{C_{1 / 2}-0} L(x, u) d \phi(u) .
$$

From the fact that for $u$ in $C_{1 / 2}-0$

$$
D_{x_{1}}^{s} D_{x_{2}}^{t} L(x, u)=\sum_{k=\max (a, 0)} \frac{(i x u)^{k-a}}{(k-a) !} \frac{\left(i u_{1}\right)^{s}\left(i u_{2}\right)^{t}}{i^{2 \omega}|u|^{2 \omega}}
$$


where $a=s+t-2 \omega$, we conclude that the partial derivatives of $L(x, u)$ with respect to $x_{1}$ and $x_{2}$ are uniformly bounded for $x$ in a bounded domain and $u$ in $C_{1 / 2}-0 . F(x)$ is therefore of class $C^{(\infty)}$ and $\nabla^{2(\omega)} F(x)=\int_{C_{1 / 2}-0} e^{i x u} d \phi(u)$.

Integrating the last integral on the right side of (16) by parts, we see that (16) can be written as

$$
\Delta_{R}(x)=\int_{C_{1 / 2}-0} e^{i x u} d \phi(u)-\left(4 \pi^{2}\right)^{-1} \int_{D} \nabla^{2(\omega)}[F(u) \lambda(u)] D_{R}(x-u) d u .
$$

Since $\nabla^{2(\omega)}[F(x) \lambda(x)]$ is of class $C^{(2 \delta)}$ and equals $\int_{C_{1 / 2}-0} e^{i x u} d \phi(u)$ on $D_{1}$, it is clear that $\Delta_{R}(x) \rightarrow 0$ uniformly for $x$ in $D_{1}$, which concludes the second part of the proof of this theorem.

For the third part of the proof, we assume $\phi(E)=0$ if 0 is not in $E$. Then $\Delta_{R}(x)$ in (16) is given by

$$
\Delta_{R}(x)=\phi(0)-\left(4 \pi^{2}\right)^{-1} \phi(0) \int_{D} \nabla^{2(\omega)}\left[\frac{\left(u_{1}+u_{2}\right)^{2 \omega}}{2 \omega ! 2^{\omega}} \lambda(u)\right] D_{R}(x-u) d u
$$

and it follows that $\Delta_{R}(x) \rightarrow 0$ uniformly for $x$ in $D_{1}$.

Observing that any non-negative additive function of a set $\phi$ can be written as a sum of three parts corresponding to the three cases of the proof considered, we see that the proof of the theorem is complete.

Before proving Theorem 1 , it will be necessary for us to reexamine the function $\mu(u)$ obtained in the first part of the proof of Theorem 5 . We do so with the following lemma.

LEMMA 5. Let $\phi$ and $\Gamma$ be two additive functions of $a$ set with the following properties:

(i) $W\left(\phi, C_{1 / 2}\right)=0$ and $W\left(\phi, C_{1}-u\right)=o(|u| \alpha), \alpha>-1$.

(ii) $\Gamma(A)=\int_{A} \gamma(u) d u$ for all Borel sets $A$, where $\gamma(u)$ is a continuous function which is $o\left(|u|^{-2(\delta+\omega)}\right), \delta$ and $\omega$ as in Theorem 5. Set $\Phi(A)=\int_{A}|u|^{-2 \omega} d \phi(u)$ and $M=\Gamma * \Phi$. Then $M(A)=\int_{A} \mu(u) d u$ where $\mu(u)$ is a continuous function and $\mu(u)=o\left(|u|^{\alpha-2 \omega}\right)$.

By Lemma 1,

$$
\begin{aligned}
M(A) & =\int_{E_{2}} \frac{\Gamma(A-p)}{|p|^{2 \omega}} d \phi(p) \\
& =\int_{E_{2}} \frac{1}{|p|^{2 \omega}} d \phi(p) \int_{E_{2}} f_{A}(u+p) \gamma(u) d u \\
& =\int_{A} d u\left[\int_{E_{2}} \frac{\gamma(u-p)}{|p|^{2 \omega}} d \phi(p)\right] .
\end{aligned}
$$

Therefore $\mu(u)=\int_{E_{2}}\left(\gamma(u-p) /|p|^{2 \omega}\right) d \phi(p)$ and $\mu(u)$ is clearly a continuous function. To show $\mu(u)=o(|u| \alpha-2 \omega)$, write 


$$
\begin{aligned}
\mu(u) & =\int_{E_{2}} \frac{\gamma(u-p)}{|p|^{2 \omega}} d \phi(p)=\int_{E_{2}} \frac{\gamma(-p)}{|p+u|^{2 \omega}} d \phi(p+u) \\
& =\int_{E_{2}-C_{|u| / 2}} \frac{\gamma(-p)}{|p+u|^{2 \omega}} d \phi(p+u)+\int_{C_{|u| / 2}} \frac{\gamma(-p)}{|p+u|^{2 \omega}} d \phi(p+u) \\
& =B_{1}+B_{2} .
\end{aligned}
$$

Now

$$
\left|B_{1}\right| \leqq O\left(\max _{p \text { in } E_{2}-C_{|u| / 2}}|\gamma(-p)|\right)=o\left(|u|^{-2(\delta+\omega))}=o\left(|u|^{\alpha-2 \omega}\right),\right.
$$

and

$$
\begin{gathered}
\left|B_{2}\right| \leqq \\
\sum_{i=1}^{[|u| / 2]+1} \int_{c_{i}-C_{i-1}} \frac{|\gamma(-p)|}{|p+u|^{2 \omega}}|d \phi(p+u)| \\
\quad+|\gamma(0)| W(\phi, 0+u)|u|^{-2 \omega} .
\end{gathered}
$$

The last expression on the right side of (20) is clearly $o\left(|u|^{\alpha-2 \omega}\right)$. For the sum on the right side of (20), we observe that the annulus $C_{i}-C_{i-1}$ with $i \leqq[|u| / 2]+1$ can be covered by less than $K i$ circles $\sigma_{n}^{i}, n=1, \cdots, m_{i}$, where $K$ is a constant independent of $i$ and $u, m_{i}<K i$, and the radius of $\sigma_{n}^{i}=1$. The following facts are true about $\sigma_{n}^{i}$ :

$$
\begin{aligned}
& \max _{p \text { in } \sigma_{n}^{i}}|\gamma(-p)|=o\left(i^{-2(\delta+\omega)}\right) . \\
& \int_{\sigma_{n}^{i}} \frac{|d \phi(p+u)|}{|p+u|^{2 \omega}}=o\left(|u|^{\alpha-2 \omega}\right) \quad \text { uniformly in } i \text { and } n .
\end{aligned}
$$

Therefore

$$
\begin{aligned}
\int_{C_{i}-C_{i-1}} \frac{|\gamma(-p)|}{|p+u|^{2 \omega}}|d \phi(p+u)| & \leqq \sum_{i=1}^{m_{i}} \max _{p \text { in } \sigma_{n}^{i}}|\gamma(-p)| \int_{\sigma_{n}^{i}} \frac{|d \phi(p+u)|}{|p+u|^{2 \omega}} \\
& \leqq K^{2-2(\delta+\omega)} o\left(|u|^{\alpha-2 \omega}\right)
\end{aligned}
$$

and the sum on the right side of $(20)$ is

$$
\leqq \sum_{i=1}^{[|u| / 2]+1} K i i^{-2(\delta+\omega)} o\left(|u|^{\alpha-2 \omega}\right)=o\left(|u|^{\alpha-2 \omega}\right) \text {. }
$$

Since both $B_{1}$ and $B_{2}$ are $o\left(|u|^{\alpha-2 \omega}\right)$, so is $\mu(u)$ and the proof of the lemma is complete.

REMARK 3. In the proof of the first part of Theorem 5 we showed that

$$
\mu(u)=\left(4 \pi^{2}\right)^{-1} \int_{E_{2}} e^{-i x u} F(x) \lambda(x) d x
$$


where $F(x)$ was the Riemann function associated with $\phi$ so chosen that $W\left(\phi, C_{1 / 2}\right)=0$ and $\lambda(x)$ was the localizing function of class $C^{[2(\delta+\omega)]}$ for two bounded closed domains one contained in the interior of the other. By Lemma 6, we can now conclude that

$$
\left(4 \pi^{2}\right)^{-1} \int_{E_{2}} e^{-i x u} F(x) \lambda(x) d x=o\left(|u|^{\alpha-2 \omega}\right) .
$$

7. Proof of Theorem 1. The proof of this theorem is divided into two parts. First we treat the case when $W\left(\phi, C_{1 / 2}\right)=0$.

Choose closed domains $D_{1}$ and $D$ contained in the interior of the fundamental square $\Omega$ such that $D^{*} \subset D_{1}^{0} \subset D_{1} \subset D^{0} \subset D \subset \Omega^{0}$. Associate with $D_{1}$ and $D$ the localizing function $\lambda_{1}(x)$ of class $C^{[2(\delta+\omega)]}$ which is 1 in $D_{1}$ and 0 outside of $D, \delta$ and $\omega$ as in Theorem 5 . Then by Remark 3

$$
\left(4 \pi^{2}\right)^{-1} \int_{E_{2}} e^{-i x u} F(x) \lambda_{1}(x) d x=o\left(|u|^{\alpha-2 \omega}\right) .
$$

We now define a function $G(x)$ periodic in each variable of period $2 \pi$ and equal to $F(x) \lambda_{1}(x)$ in $\Omega$. Developing $G(x)$ in a Fourier series and designating this series by $\mathfrak{S}[G]$, we have

$$
\subseteq[G]=a_{0}+(-1)^{\omega} \sum_{m}^{\prime} a_{m}|m|^{-2 \omega} e^{i m x}
$$

where ' indicates the omission of 0 . By (21), $a_{m}=o\left(|m|^{\alpha}\right)$. Since $\alpha-2 \omega$ $=\alpha-2([\alpha / 2]+2)=-(2+\nu)$ where $\nu>0$, we have that the above series converges absolutely and uniformly to $G(x)$.

Designating by $\Lambda$ the set of all lattice points $m=\left(m_{1}, m_{2}\right)$ in the plane, we define an additive function of sets $\theta$ in the following manner:

$\theta(m)=a_{m}$ for $m \neq 0, \theta(0)=0$,

$\theta\left(\cup_{m}\right)=\sum \theta(m)$ for all finite union of lattice points,

$\theta(A)=\theta(A \cap \Lambda)$ for any bounded Borel set $A$.

It is then clear that $W\left(\theta, C_{1}-u\right)=o\left(|u|^{\alpha}\right), W\left(\theta, C_{1 / 2}\right)=0, \int_{C_{R}} e^{i x u} d \theta(u)$ $=\sum_{|m| \leqq R}^{\prime} a_{m} e^{i m x}$, and that the Riemann function associated with $\int_{E_{2}} e^{i x u} d \theta(u)$ is

$$
(-1)^{\omega} \sum_{m} a_{m}|m|^{-2 \omega} e^{i m x}=G(x)-a_{0} .
$$

Let $\lambda(x)$ be the localizing function of class $C^{[2(\delta+\omega)]}$ which is 1 in $D^{*}$ and 0 outside of $D_{1}$. Then by Theorem 5 ,

$$
\sum_{|m| \leqq R}^{\prime} a_{m} e^{i m x}-\left(4 \pi^{2}\right)^{-1} \int_{D_{1}}\left[G(u)-a_{0}\right] \lambda(u) \nabla^{2(\omega)} D_{R}(x-u) d u
$$

is uniformly summable $(C, \alpha+1)$ to zero for $x$ in $D^{*}$. But clearly $\int_{D_{1}} a_{0} \lambda(u) \nabla^{2(\omega)} D_{R}(x-u)$ converges to zero uniformly for $x$ in $D^{*}$. Further- 
more $G(x)=F(x)$ in $D_{1}$. We therefore conclude from (23) that

$$
\sum_{|m| \leqq R}^{\prime} a_{m} e^{i m x}-\left(4 \pi^{2}\right)^{-1} \int_{D_{1}} F(u) \lambda(u) \nabla^{2(\omega)} D_{R}(x-u) d u
$$

is uniformly summable $(C, \alpha+1)$ to zero for $x$ in $D^{*}$. This fact coupled with Theorem 5 concludes the proof to the first part of the theorem.

REMARK 4 . We point out for use in the proof of Theorem 3 that in the case $W\left(\phi, C_{1 / 2}\right)=0$ the series whose circular partial sums are uniformly equisummable $(C, \alpha+1)$ with $\int_{C_{R}} e^{i x u} d \phi(u)$ in $D^{*}$ has no constant term. Designating the series by $\sum^{\prime} a_{m} e^{i m x}$, we see from (22) that the Riemann function associated with this series is $(-1)^{\omega} \sum^{\prime} a_{m}|m|^{-2 \omega} e^{i m x}=G(x)-a_{0}$ and that $G(x)-a_{0}$ differs from the Riemann function associated with $\int_{E_{2}} e^{i x u} d \phi(u)$ in $D^{*}$ by a constant.

For the second part of the proof of Theorem 1, we assume that $W(\phi, A)$ $=0$ if $A$ is a Borel set lying outside of $C_{1 / 2}$. Then $g(x)=\int_{C_{1 / 2}} e^{i x u} d \phi(u)$ is a function in $C^{(\infty)}$, and $\lambda(x) g(x)$ is a function in class $C^{[2(\delta+\omega)]}$, where $\lambda(x)$ is the localizing function for $D^{*} \subset D_{1}^{0} \subset D_{1} \subset \Omega^{0}$ of the first part of the proof of this theorem. Set $h(x)=\lambda(x) g(x)$ in $\Omega$ and make $h(x)$ periodic of period $2 \pi$ in each variable. Then $h(x)$ can be expanded in a Fourier series $\sum_{m} b_{m} e^{i m x}$ where $b_{m}=o\left(|m|^{-2(\delta+\omega)}\right)$. This series, however, converges absolutely and uniformly to $h(x)$ and therefore to $g(x)$ for $x$ in $D^{*}$. Therefore $\int_{C_{R}} e^{i x u} d \phi(u)-\sum_{|m| \leqq R} b_{m} e^{i m x}$ converges uniformly to zero for $x$ in $D^{*}$, which fact proves the second part of this theorem.

Observing that any additive function of a set $\phi$ can be decomposed into two set functions, one corresponding to each part of the proof, we see that the proof of the theorem is complete.

8. Proof of Theorem 2. By Remark 2, after setting $g(u)=(-1)^{\omega}|u|^{2 \omega} \mu(u)$, we see that the theorem is proved in case $W\left(\phi, C_{1 / 2}\right)=0$.

Let us assume that $W(\phi, A)=0$ if $A$ is a Borel set lying outside of $C_{1 / 2}$. Then $h(x)=\int_{C_{1 / 2}} e^{i x u} d \phi(u)$ is a function in class $C^{(\infty)}$. Let $D$ be a bounded closed domain containing $D_{1}$ in its interior and let $\lambda(x)$ be the localizing function for $D_{1}$ and $D$ of class $C^{(4)}$. Then $h(x) \lambda(x)$ is a function of class $C^{(4)}$. Setting $g(u)=\left(4 \pi^{2}\right)^{-1} \int_{E_{2}} e^{-i x u} h(x) \lambda(x) d x$, we see that $g(u)=o\left(|u|^{-4}\right)$ and that $\int_{C_{R}} g(u) e^{i x u} d u$ is uniformly convergent to $\int_{C_{1 / 2}} e^{i x u} d \phi(u)$ for $x$ in $D_{1}$.

Observing that a general $\phi$ can be decomposed into two additive functions of a set, one corresponding to each part of the proof, we see that the theorem is proved.

9. Proof of Theorem 3. Let us assume first that $c(u)=0$ if $u$ is in $C_{1 / 2}$. Then the additive function of a set $\phi$ defined by $\phi(A)=\int_{A} c(u) d u$ for any bounded Borel set $A$ is such that $W\left(\phi, C_{1 / 2}\right)=0$ and $W\left(\phi, C_{1}-u\right)=o\left(|u|^{-\epsilon}\right)$. Let $F(x)=-\int_{E_{2}} e^{i u x} c(u)|u|^{-2} d u$ be the Riemann function associated with the trigonometric integral $\int_{E_{2}} e^{i x u} d \phi(u)$. We shall show that $F(x)$ is in class $C^{(2)}$ and that $\nabla^{2} F(x)=f(x)$. 
For given any point $\bar{x}$, consider the closed disc $C_{1}+\bar{x}$. By Remark 4 in the proof of Theorem 1 , there is a trigonometric series $\sum^{\prime} a_{m} e^{i m x}$ with coefficients $a_{m}=o\left(|m|^{-\epsilon}\right)$ such that $\int_{C_{R}} e^{i x u} c(u) d u-\sum_{|m| \leqq R}^{\prime} a_{m} e^{i m x}$ is $(C, 1)$ summable to zero uniformly for $x$ in $C_{1}+\bar{x}$ and such that $G(x)-a_{0}$, the Riemann function associated with this series, differs from $F(x)$ in $C_{1}+\bar{x}$ by a constant. Also it is clear that $\sum_{|m| \leqq R}^{\prime} a_{m} e^{i m x}$ is summable $(C, 1)$ to $f(x)$ in $C_{1}+\bar{x}$. By [8, Theorem 1, and 9, Lemma 2] the generalized Laplacian of $G(x), \Delta_{1} G(x)$, is such that $\Delta_{1} G(x)=f(x)$ in $C_{1}+\bar{x}$. So in particular $\Delta_{1} F(\bar{x})=f(\bar{x})$. Since $\bar{x}$ was an arbitrary point we conclude that $\Delta_{1} F(x)=f(x)$ for all $x$. Furthermore since $c(u)|u|^{-2}$ is in $L_{1}$ on $E_{2}$, we have that $F(x)$ is continuous. These last two facts plus the fact that $f(x)$ satisfies a Lipschitz condition of order $\alpha$ on every bounded domain enables us to conclude that $F(x)$ is in class $C^{(2)}$ and that $\nabla^{2} F(x)=f(x)$. (See [6, Theorem 1] and [5, pp. 289-296].)

By [3, Theorem 1], $\left(4 \pi^{2}\right)^{-1} \int C_{R} F(x) e^{-i x u} d u$ is $(C, 1)$ summable to $-c(u)|u|^{-2}$ almost everywhere. But by Green's second identity, for $u \neq 0$,

$$
\begin{aligned}
\int_{C_{R}} F(x) e^{-i x u} d u= & -\int_{C_{R}}|u|^{-2} f(x) e^{-i x u} d x \\
& -\int_{0}^{2 \pi} F(R \cos \theta, R \sin \theta) \partial\left(|u|^{-2} e^{-i R|u| \cos \left(\theta-\theta_{1}\right)}\right) / \partial R R d \theta \\
& +\int_{0}^{2 \pi}|u|^{-2} e^{-i R|u| \cos \left(\theta-\theta_{1}\right)} \partial F(R \cos \theta, R \sin \theta) / \partial R R d \theta
\end{aligned}
$$

where $u=\left(|u| \cos \theta_{1},|u| \sin \theta_{1}\right)$.

Calling the last two integrals on the right side of (25) $I_{R}^{a}$ and $I_{R}^{b}$ respectively, we shall now show that both $I_{R}^{a}$ and $I_{R}^{b}$ are $(C, 1)$ summable to zero. For we observe that

$$
\begin{aligned}
\left|R^{-2} \int_{0}^{R} I_{r}^{a} r d r\right| & =\left.R^{-2}\left|\int_{C_{R}}\right| x\left|F(x) \cos \left(\theta-\theta_{1}\right)\right| u\right|^{-1} e^{-i x u} d x \mid \\
& \leqq|u|^{-1} R^{-2} \int_{C_{R}}|x||F(x)| d x .
\end{aligned}
$$

Now since $c(u)|u|^{-2}$ is in $L_{2}$ on $E_{2}$, we have by the Plancherel theorem that $F(x)$ is in $L_{2}$ and consequently that $R^{-2} \int_{C_{R}}|x||F(x)| d x$ tends to zero as $R$ tends to infinity, for

$$
\begin{aligned}
\int_{C_{R}}|x||F(x)| d x \leqq & \int_{C_{R_{0}}}|x||F(x)| d x \\
& +\left[\int_{C_{R}-C_{R_{0}}}|x|^{2} d x\right]^{1 / 2}\left[\int_{E_{2}-C_{R_{0}}}|F(x)|^{2} d x\right]^{1 / 2} .
\end{aligned}
$$

$I_{R}^{a}$ is, therefore, $(C, 1)$ summable to zero. 
To show that $I_{R}^{b}$ is $(C, 1)$ summable to zero, we observe that

$$
\begin{aligned}
& R^{-2} \int_{0}^{R} I_{r}^{b} r d r \\
& \quad=R^{-2}|u|^{-2} \int_{0}^{2 \pi} d \theta \int_{0}^{R} r^{2} e^{-i r|u| \cos \left(\theta-\theta_{1}\right)} \frac{\partial F}{\partial r}(r \cos \theta, r \sin \theta) d r .
\end{aligned}
$$

After integrating by parts, we see that the right side of (26) becomes

$$
\begin{aligned}
|u|^{-2} R^{-2} \int_{0}^{2 \pi} F(R \cos \theta, R \sin \theta) e^{-i R|u| \cos \left(\theta-\theta_{1}\right)} R^{2} d \theta \\
\quad-|u|^{-2} R^{-2} \int_{C_{R}} F(x)\left[2 e^{-i x u}-i|x||u| \cos \left(\theta-\theta_{1}\right) e^{-i x u}\right] d x .
\end{aligned}
$$

Since $F(x)$ is the Fourier transform of a function in $L_{1}, F(x)=o(1)$ as $|x| \rightarrow \infty$. Consequently the first expression in (27) tends to zero as $R \rightarrow \infty$. The second expression in (27) tends to zero as $R \rightarrow \infty$ from considerations similar to those involved in the discussion concerning $I_{R}^{a}$.

We conclude, therefore, from (25) that, under the assumption $c(u)=0$ for $|u| \leqq 1 / 2$, for almost every $u,\left(4 \pi^{2}\right)^{-1} \int_{E_{2}} e^{-i x u} f(u) d u$ is circularly summable $(C, 1)$ to $c(u)$ for $|u|>1 / 2$ and to 0 for $|u| \leqq 1 / 2$.

Now given a general $c(u)$, set $f_{1}(x)=\int_{C_{1 / 2}} e^{i x u} c(u) d u$ and notice that $f_{1}(x)$ is in class $C^{(\infty)}$. Then $\left(4 \pi^{2}\right)^{-1} \int_{E_{2}} f_{1}(x) e^{-i x u} d u$ is, for almost every $u,(C, 1)$ circularly summable to $c(u)$ for $|u| \leqq 1 / 2$ and to zero for $|u|>1 / 2$. Furthermore, by the above proof, for almost every $u,\left(4 \pi^{2}\right)^{-1} \int_{E_{2}}\left[f(x)-f_{1}(x)\right] e^{-i x u} d u$ is $(C, 1)$ circularly summable to $c(u)$ for $|u|>1 / 2$ and to 0 for $|u| \leqq 1 / 2$, which fact concludes the proof to the theorem.

10. Extensions to higher dimensions. Designating the closed $k$-dimensional sphere with radius at the origin by $C_{R}^{(k)}$, we give the following $k$-dimensional analogue to Theorem 1 without proof:

THEOREM 6. Let $T=\int_{E_{k}} e^{i x u} d \phi(u)$ be a k-dimensional trigonometric integral with $W\left(\phi, C_{1}^{(k)}-u\right)=o\left(|u|^{\alpha}\right), \alpha>-(k-1), k \geqq 2$. Let $D^{*}$ be a closed domain contained in the interior of the $k$-dimensional fundamental cube $\Omega$. Then there exists a k-dimensional trigonometric series $S=\sum a_{m} e^{i m x}$ with coefficients $a_{m}$ $=o\left(|m|^{\alpha}\right)$ such that $\int_{C_{R}(k) e^{i x u}} d \phi(u)-\sum_{|m| \leqq R} a_{m} e^{i m x}$ is summable $(C, \alpha+k-1)$ to zero, uniformly for $x$ in $D^{*}$.

A similar $k$-dimensional analogue exists for Theorem 2. It is not apparent, however, that Theorem 3 can be extended to $k$-dimensional space using the techniques of this paper as investigation of the proof demonstrates.

\section{REFERENCES}

1. L. D. Berkovitz, On double trigonometric integrals, Trans. Amer. Math. Soc. vol. 73 (1952) pp. 345-372. 
2. - Circular summation and localization of double trigonometric series, Trans. Amer. Math. Soc. vol. 70 (1951) pp. 323-344.

3. S. Bochner, Summation of multiple Fourier series by spherical means, Trans. Amer. Math. Soc. vol. 40 (1936) pp. 175-207.

4. M. Cheng, Uniqueness of multiple trigonometric series, Ann. of Math. vol. 52 (1950) pp. 403-416.

5. N. M. Gunther, La théorie du potentiel et ses mathematiques applications aux problèmes fondamentaux de la physique, Paris, 1934.

6. W. Rudin, Integral representation of continuous functions, Trans. Amer. Math. Soc. vol. 68 (1950) pp. 279-287.

7. S. Saks, Theory of the integral, Warsaw, 1937.

8. V. L. Shapiro, Circular summability $C$ of double trigonometric series, Trans. Amer. Math. Soc. vol. 76 (1954) pp. 223-233.

9. - An extension of results in the uniqueness theory of double trigonometric series, Duke Math. J. vol. 20 (1953) pp. 359-366.

10. A. Zygmund, On trigonometric integrals, Ann. of Math. vol. 48 (1947) pp. 393-440.

RUTGERS UNIVERSITY,

NEW BRUNSWICK, N. J. 